parents of children aged 0 to 16 years old and by young people up to the age of 18 years.

\section{P10 AN AUDIT TO ASSESS THE PRESCRIBING OF ANALGESIA IN CHILDREN WHO PRESENT WITH PAIN CRISIS DUE TO SICKLE CELL DISEASE (SCD)}

Masuma Dhanji* . Luton and Dunstable Hospital

\subsection{6/archdischild-2020-NPPG.19}

Aim To assess the prescribing of analgesia to manage pain crises in children with SCD. This was to establish whether the Trust was meeting national and local standards. Prompt pain control is essential to reduce length of stay and further complications. ${ }^{1}$

Standards

- $100 \%$ of admissions will be prescribed regular paracetamol and non-steroidal anti-inflammatory drugs (NSAIDs) at the recommended frequency unless contraindicated in accordance with national guidance. ${ }^{2} 3$

- $100 \%$ of admissions will be prescribed appropriate doses of analgesia with consideration to weight and age in accordance with local policy.

Method The audit was registered with the Trust's audit committee. A list of paediatric patients with the diagnosis of SCD was sought from paediatricians with an interest in haematology. A data collection form was created. Data was collected retrospectively over a one-year period. A total of 60 admissions were reviewed to check whether analgesia was prescribed regularly at the recommended frequency, and at the correct dose. Results were analysed using descriptive statistical analysis. Exclusion criteria included patients with hospital admissions under 24 hours.

Results A total of 55 admissions were included in the final sample. The audit showed the Trust was non-adherent to both standards assessed. A total of 45\% (95\% CI [31.9\%, 58.1\%]) of admissions were prescribed regular analgesia. A total of $78 \%$ (95\% CI [67.9\%, 88.9\%] of admissions were prescribed appropriate doses of analgesia. Two main reasons were found as to why analgesia was prescribed at the incorrect dose. This was due to incorrect weights recorded on the electronic system $(n=4)$ and doses based on age only $(n=8)$.

Conclusion The results show prescribers are familiar with the correct doses of analgesia but fail to prescribe analgesia regularly. This highlights an opportunity for education and training in the management of pain crisis in SCD. One recommendation includes development of an integrated care pathway booklet for paediatric patients presenting with pain crisis due to SCD. Integrated care pathway booklets have been implemented for other conditions such as cystic fibrosis yielding positive outcomes. The results have highlighted key issues surrounding the electronic prescribing system such as out-of-date weights remaining on the system unless updated, and default treatment protocols. The electronic prescribing system requires refinement for use within paediatrics. One suggestion includes compulsory weight field on admission. Limitations of this audit included small sample size. There was a lack of data to make suggestions based on different ages.

\section{REFERENCES}

1. Rees D, Olujohungbe $A$, Parker $N$, et al. Guidelines for the management of the acute painful crises in sickle cell disease. Br J Haemato 2003;120:744-752.
2. National Institute for Health and Care Excellence (2012) Sickle cell disease: managing acute painful episodes in hospital. NICE Guideline (CG143).

3. Paediatric Formulary Committee. BNF for Children (2018-2019). London: BMJ Group, Pharmaceutical Press, and RCPCH Publications; (2018).

4. General Hospital (2015) Management of sickle cell disease in paediatric patients (CG377).

\section{P11 ARE LOW MOLECULAR WEIGHT HEPARINS BEING INITIATED, MONITORED AND SUBSEQUENTLY ADJUSTED APPROPRIATELY FOR PAEDIATRIC PATIENTS?}

Colleen Duffy* . Imperial College Healthcare NHS Trust, London

\subsection{6/archdischild-2020-NPPG.20}

Aim Heparin is used in patients who require anticoagulation for treatment or prevention of thrombosis. Much of the evidence for anticoagulation with both unfractionated and low molecular weight heparin (LMWH) is derived from adult practice.

This audit aimed to evaluate the accuracy of tinzaparin dosing and monitoring, and thus the provision of appropriate anticoagulation for treatment and prevention of thrombosis in paediatric patients. This was in line with trust clinical guidelines: 'Low molecular weight heparin guideline: paediatrics (treatment and prophylaxis)'. ${ }^{1}$

Method Paediatric patients prescribed Tinzaparin between November 2017 and December 2018 were retrospectively identified from finance reports. Patient notes, which documented Tinzaparin indication, dosing and monitoring parameters (Anti-Xa levels) were accessed. Findings were recorded in a data collection questionnaire, derived from set standards, to identify if the corresponding local guidelines had been adhered to. ${ }^{1}$ Subsequent statistical analysis was used to highlight trends within the data collection.

Results $88 \%$ (21/24) of paediatric patients were dosed accurately according to Tinzaparin indication; treatment or prophylaxis and patient weight as per guidelines. One anomaly was dosed according to local guidelines for adult patients, whilst a second and third were initiated on prophylactic rather than treatment dosing. Only $11 \%(3 / 24)$ of paediatric patients had their Anti-Xa level recorded at the correct time interval of 4 hours post dose. Evaluation of this data confirmed that for prophylactic regimens Anti-Xa levels were recorded in $7 \%$ $(1 / 16)$ of patients, compared to $33 \%$ (3/8) for treatment regimens. Although Anti-Xa levels were recorded throughout $100 \%(8 / 8)$ of tinzaparin treatment regimens, 66\% (5/8) failed to be recorded within four hours post first and second dose; a guideline requirement. These 'random' Anti-Xa levels commonly lay outside of the desired Anti-Xa level range highlighted in the guideline and subsequent dose adjustment meant that dosing regimens deviated from guidelines in an attempt to get the Anti-Xa levels within range. For regimens that lay outside the desired range but that were then adjusted in accordance with a dose adjustment tool within the guideline, all patients achieved the desired range efficiently and effectively, confirming that following the guideline achieves desirable results.

Conclusions It was clear that Tinzaparin was initiated appropriately in the majority of paediatric patients in accordance with patient age and weight, that an attempt was made to monitor patients receiving a treatment dose regimen and that some effort was made to maintain these levels within the desired range. The main issue raised by this audit was the 
lack of adequate Tinzaparin monitoring throughout prophylactic dosing, thus highlighting an opportunity to educate and communicate the guideline to health care professionals within this field of practice to encourage effective treatment and prophylaxis of thrombosis. Raising awareness for the need of adequate documentation within patient notes to explain omitted dosing would also guide healthcare professionals involved in patient care to make informed decisions and avoid unnecessary alterations to treatment plans.

\section{REFERENCES}

1. Lumb P, Fletcher P. 2017. Low Molecular Weight Heparin Guideline: Paediatrics (Treatment and Prophylaxis). Imperial College NHS Trust.

2. Medicines.org.uk. 1997. Tinzaparin sodium Syringe $10,000 \mathrm{IU} / \mathrm{ml}$ - Summary of Product Characteristics (SmPC) - (eMC). [online] Available at: https://www.medicines.org.uk/emc/product/2022/smpc [Accessed 19 Mar. 2019]

\section{P12 EVALUATION OF ENERGY AND PROTEIN INTAKE IN NEONATES USING SCAMP REGIMEN}

${ }^{1}$ Sian Gaze*, ${ }^{2}$ Chidubem Okoye, ${ }^{2}$ Graham Davies. ${ }^{1}$ Evelina London Children's Hospital; ${ }^{2}$ King's College University London

10.1136/archdischild-2020-NPPG.21

Aims Our neonatal unit recently updated their parenteral and enteral feeding guideline and started using a standardised parenteral nutrition regimen (SCAMP). ${ }^{1}$

The aim of this study was to observe the amount of energy and protein that was being delivered to patients using the SCAMP regimen and to evaluate whether it met the recommendations made by ESGPHAN in terms of energy and protein intake. ${ }^{2}$

Methods A data collection form was designed using Excel. Patients were identified using BadgerNet digital software. All required patient parameters were recorded from Badgernet. Data was anonymised and the Excel spreadsheet was password protected. A single investigator collected data over a 28 day period in October/November 2018. Only patients on the SCAMP regimen were included in the study. Patient data was collected from first day of SCAMP regimen until baby was discharged, or ceased parenteral nutrition.

Results

- 22 patients were identified for inclusion in the study. Of these, $17 / 22(77 \%)$ weighed $<1 \mathrm{~kg}$.

- Majority of babies (20/22; 91\%) were aged < 31 weeks corrected gestational age when SCAMP was initiated.

- Majority of babies were on the SCAMP regimen due to prematurity; 4/22 (18\%) had a surgical condition, that required $\mathrm{PN}$ to be initiated.

- 1 patient had a complex congenital cardiac anomaly.

- Average length of time babies remained on SCAMP was 19 days (range 5-28 days).

- Majority of babies were enterally fed using breastmilk. Some babies were on alternative formula feeds.

- Mean amount of energy delivered to each patient was calculated. The amount of energy delivered increased daily over the first week, and by day 10 of life had reached the target range (110-135 kcal/kg/day). Recommended energy intake was then maintained for the rest of the 28-day study period.

- Target amount of protein intake varied for babies weighing $<1 \mathrm{~kg}$ and $>1 \mathrm{~kg}$. For babies $<1 \mathrm{~kg}$, a gradual increase occurred over the first 5 days of life. Recommended protein intake was met between days 5-10 of life, then there was a gradual decline.

- For babies weighing between 1-1.8 kg, the recommended protein intake was achieved within $24-48$ hours. Higher than recommended amounts of protein were being delivered between days 5-10 of life. Mean protein intake remained within ESPGHAN recommendations (3.5-4 g/ kg/day) during weeks 3 and 4 of life.

The results are encouraging and demonstrate that neonates are managing to achieve the recommended amounts of energy intake from day 10 of life.

Limitations This study focused solely on energy and protein intake - it did not include observations of growth. Future studies should consider looking at more patient-focused outcomes.

Conclusions SCAMP regimen is delivering the recommended amounts of energy for babies on the neonatal unit - target levels are achieved by day 10 of life.

- Future work should focus on observing growth in babies on SCAMP

- Earlier introduction of breast-milk fortifier may be helpful to increase protein intake in babies $<1 \mathrm{~kg}$ - potential benefits need to be evaluated against risk of adverse effects.

\section{REFERENCES}

1. Morgan C, Herwitker S, Badhawi I, et al. SCAMP: standardised, concentrated additional macronutrients, parenteral nutrition in very preterm infants: a phase IV randomised, controlled exploratory study of macronutrient intake, growth and other aspects of neonatal care. BMC Pediatrics 2011;11:53.

2. Koletzo B, Goulet 0 , Hunt J, et al. Guidelines on paediatric parenteral nutrition of the European society of paediatric gastroenterology, hepatology and nutrition (ESPGHAN) and the European society for clinical nutrition and metabolism (ESPEN), supported by the European society of paediatric research (ESPR). I Pediatr Gastroenterol Nutr 2005;41 (Suppl 2):S1-87.

\section{P13 OPTIMISING PAEDIATRIC INTRAVENOUS FLUID MANAGEMENT PLANS: A QUALITY IMPROVEMENT PROJECT}

${ }^{1}$ Jenny Gray ${ }^{*},{ }^{2}$ Susie Gage. ${ }^{1}$ University Hospitals Bristol; ${ }^{2}$ Bristol Children's Hospital

\subsection{6/archdischild-2020-NPPG.22}

Introduction Intravenous (IV) maintenance fluids are often prescribed post-surgery when enteral routes are contraindicated. Serious consequences have been documented when poor fluid management has occurred, as highlighted in the National Patient Safety Alert (NPSA) 22; reducing the risk of hyponatraemia; when administering IV fluids to children. ${ }^{1}$ In response to this, the National Institute for Health and Care Excellence (NICE) published their guidance in December 2015 regarding IV fluids in children. ${ }^{2}$ Based on NICE recommendations, a pan hospital fluid guidance was produced. Within the NICE and hospital's own guideline it states that there should be a daily fluid management plan documented. It has been well recognised that this daily fluid management plan was not routinely been completed; hence showing non-adherence to our hospital policy and NICE recommendations.

Aims Primary aim was to improve the documentation of the daily fluid management plan; aimed at the medical staff and the secondary aim was to improve the monitoring requirements of IV fluids and documentation of these; largely aimed at the nursing staff. 